\title{
Resource Allocation for Licensed/Unlicensed Carrier Aggregation MIMO Systems
}

\author{
Christos G. Tsinos *, Fotis Foukalas *, Theodoros A. Tsiftsis *† \\ *Industrial Systems Institute, Athena Research and Innovation Center, 26504 Patras, Greece \\ $\dagger$ School of Engineering, Nazarbayev University, Astana 01000, Kazakhstan \\ Email: tsinos@isi.gr, foukalas@isi.gr, theodoros.tsiftsis@nu.edu.kz
}

\begin{abstract}
In this paper a novel Carrier Aggregation (CA) scheme is proposed for downlink MIMO LTE-A Systems. The proposed approach achieves increased transmission rates by establishing the communication links via both licensed and unlicensed bands without generating or experiencing interference to/from the users of the latter bands. To that end, a rate optimization problem is defined and solved subject to the previous zero interference constraints, a total power constraint and a maximum number of aggregated bands constraint. It turns out that the previous problem is a Mixed Integer Non Linear Programming (MINLP) one that requires an exhaustive search procedure in order to be solved. To tackle this, an optimal low complexity method is proposed based on the Lagrange dual decomposition. The performance of the original (MINLP) and the low-complexity proposed techniques is verified via indicative simulations.
\end{abstract}

Keywords-Carrier Aggregation, MIMO, LTE-A, Unlicensed Transmission, Interference Nulling, Resource Allocation, Carrier Selection.

\section{INTRODUCTION}

Carrier aggregation (CA) [1] is one of the key features of LTE-Advanced (LTE-A) systems. According to the CA approach, the user equipments (UEs) can access a wider transmission bandwidth by using $\mathrm{CA}$ to aggregate a number of individual component carriers (CCs) shaping heterogeneous contiguous and non-contiguous frequency bands. These bands can be also unlicensed to the system and therefore a transmission scheme should include sophisticated interference management techniques, according to 3GPP standards [2]. Several new challenging issues arise in radio resource management (RRM) framework for LTE-A systems supporting multiple$\mathrm{CC}$ operation, including the selection of the optimal subset of carriers and or the optimal power allocation that maximize the rate of a UE etc. The performance of CA over deploying independent carriers is investigated from different aspects in [3]-[6]. Resource allocation schemes are also important for the efficient implementation of a CA scheme. A cross layer approach for carrier selection and power control for the uplink of a CA system is examined in [7]. A greedy algorithm for efficient resource allocation for the downlink case is proposed in [8]. The theoretical analysis and the experimental results in these works show that CA can significantly enhance the system capabilities in user accommodation and throughput.

It is noteworthy that to the best of our knowledge, existing works consider only the aggregation of licensed bands, and therefore are not suitable to schemes that employ also unlicensed ones due to the interference from/to them as it was discussed above. Furthermore, little work has been done concerning the application of the CA concept in MIMO systems [9]-[13]. Existing works are limited to the experimental evaluation of simple approaches that do not fully exploit the capabilities offered by a multi-antenna transceiver structure. MIMO systems can provide increasing multiplexing gains via available spatial degrees of freedom and efficient methods to mitigate the interference generated by transmissions of multiple users over the unlicensed bands. Therefore, optimizing the transmission parameters of a MIMO CA system is not a trivial task that needs to be addressed by efficient methods as well.

Motivated by the above, we propose an innovative resource allocation scheme for multi-user MIMO LTE-A systems that are capable of aggregating both licensed and unlicensed bands. The proposed approach aims at maximizing the sum rate of the LTE-A system by exploiting its MIMO structure for nullingout the interference in the unlicensed bands and allocating optimally the CCs and the power to the available UEs. In order to satisfy the previous requirements, the sum rate maximization problem is solved subject to a total transmission power constraint, a constraint on the total number of the aggregated bands (licensed and unlicensed) and constraints related to the interference the LTE-A systems experience or generate to other terminals in the unlicensed bands. Note that to the best of our knowledge this is the first time that a resource allocation problem is examined considering both licensed and unlicensed bands for multi-user MIMO systems. This is considered very practical solution for the LTE-A system, if we take into account the last developments on the licensed assisted access scenario within the framework of 3GPP. The problem under consideration lies in the category of MINLP ones and thus, it requires an exhaustive search procedure in order to be solved. Therefore we were motivated to seek for lowcomplexity solutions. It turns out that by applying a Lagrange dual decomposition method, similar to the approach followed in[14]-[15] for resource allocation in single antenna OFDM systems, the original problem is equivalent to a number of parallel simpler optimization ones and thus, a corresponding low-complexity algorithm is also proposed.

Note, that in general, the RA problems in unlicensed bands are already examined in literature in the context of Cognitive Radio (CR) OFDM systems [16]-[17]. In these works the joint sub-carrier and power allocation for a number of Secondary Users (SUs) is examined under the presence of Primary Users (PUs) that are licensed to these sub-carriers. On the contrary, 
in the present paper we consider the case where the licensed system share a number of both licensed and unlicensed bands compatible to the LAA 3GPP. Furthermore, in existing CR related problems a typical underlay paradigm is considered where the RA problem is solved subject to a constraint on the total interference to the PUs. Here, the RA problem is solved subject to zero interference constraints to/from the PU in the unlicensed bands. Thus, both the PUs' and the SUs' transmissions are protected from the interference due to the simultaneous sub-carrier use. Finally, the proposed RA approach follows the 3GPP regulations [1] and as a result, in the corresponding optimization problem the LTE-A system includes constraints that permit the maximum number of allocated RBs per UE which perplex further the derivation of the solution and they are not taken into account in the existing CR approaches.

The rest of the manuscript is organized as follows. In Section II the system model description is given. In Section III the proposed optimal resource allocation technique is presented. Section V presents a number of indicative simulations and Section VII concludes the work.

\section{SySTEM DESCRIPTION}

\section{A. Preliminaries}

Let us consider a typical downlink scenario in a $N_{U}$ UE LTE-A system that applies a CA scheme exploiting both licensed and unlicensed CCs in order to optimize its transmission [18] . Each UE forms a $R_{s} \times T_{s}$ MIMO system with the eNodeB terminal. The extension to UEs of different number of antennas is straightforward. Each one of the available $\mathrm{CCs}$ is organized in Resource Blocks (RBs) which is the basic scheduling unit following the 3GPP standard. Each RB includes with its turn $N_{c}=12$ sub-carriers.

Let us further assume that a UE can aggregate a maximum of $K_{\max } \mathrm{RBs}$ out of $K_{U}$ un-licensed and $K_{L}$ licensed ones. The unlicensed bands are occupied by a number of MIMO users, i.e. Wi-Fi transceivers, each one having $R_{p}$ receive and $T_{p}$ transmit respectively. From now and on, we adopt the CR terminology and refer to that users via the term Primary User (PU) for clarity. Each of the unlicensed RBs have at most one active PU each timeslot with probability $\mathcal{P}_{A}$. Extension to cases having different activity probabilities and/or multiple active PUs per RBs are straightforward, though they are not shown here for the simplicity of analysis. Finally it is assumed that each one of the available RBs (both the licensed and the unlicensed ones) can be occupied by at most one UE each time following the 3GPP standards [1].

\section{B. Signal Modeling}

Let us move now to the actual description of the system. The input-output relationship of the $l-t h\left(1 \leq l \leq N_{U}\right)$ user in a licensed RB $k\left(1 \leq k \leq K_{L}\right)$ at a time index $n$ $(1 \leq n \leq N)$ within a timeslot is given by

$$
\mathbf{y}_{l k n}=\mathbf{T}_{l k}^{H} \mathbf{H}_{l k} \mathbf{S}_{l k} \sqrt{\mathbf{P}_{l k}} \mathbf{x}_{l k n}+\mathbf{T}_{l k}^{H} \mathbf{z}_{l k n}
$$

where $\mathbf{H}_{l k}$ is the $R_{s} \times T_{s}$ channel matrix modeled as $\mathcal{C} N\left(\mathbf{0}_{R_{s} \times T_{s}}, \sigma_{h}^{2} \mathbf{I}_{R_{s} \times T_{s}}\right)$ which is assumed to be fixed within the timeslot following a quasi-static fading model, $\sigma_{h}^{2}$ is the channel variance, $\mathbf{I}_{R_{s} \times T_{s}}$ is the $R_{s} \times T_{s}$ identity matrix and $\mathbf{0}_{R_{s} \times T_{s}}$ is the $R_{s} \times T_{s}$ zero entries matrix, $\mathbf{S}_{l k}$ and $\mathbf{T}_{l k}$ are the $L_{k}^{s} \times T_{s}$ pre- and $R_{s} \times L_{k}^{s}$ post-coding matrices, $\mathbf{P}_{l k}$ is the diagonal $L_{k}^{s} \times L_{k}^{s}$ power allocation matrix, $L_{k}^{s}$ is the number of transmitted streams, $\mathbf{x}_{l k n}$ is the $L_{k}^{s} \times 1$ transmitted symbols vector with covariance matrix $\mathbf{R}_{x}=\mathbf{I}_{L}$ (we follow this assumption for simplicity and without any loss of generality), $\mathbf{y}_{l k n}$ is the $R_{s} \times 1$ received symbols vector and $\mathbf{z}_{l k n}$ is the $R_{s} \times 1$ white noise random variable vector modeled as $\mathcal{C} N\left(\mathbf{0}_{R_{s}}, \sigma_{z}^{2} \mathbf{I}_{R_{s}}\right)$ with $\sigma_{z}^{2}$ the noise variance.

In an unlicensed $\mathrm{RB} k\left(K_{L} \leq k \leq K_{L}+K_{U}\right)$, eq.(1) is re-written as

$\mathbf{y}_{l k n}=\mathbf{T}_{l k}^{H} \mathbf{H}_{l k} \mathbf{S}_{l k} \sqrt{\mathbf{P}_{l k}} \mathbf{x}_{l k n}+\mathbf{T}_{l k}^{H} \mathbb{1}_{k}\left\{\mathbf{G}_{l k} \mathbf{d}_{k n}\right\}+\mathbf{T}_{l k}^{H} \mathbf{z}_{l k n}$,

where the $R_{s} \times L_{p}$ matrix $\mathbf{G}_{l k}^{H}$ incorporates the $R_{p} \times T_{p}$ channel matrix between the PU transmitter and the $l-t h$ UE, the $T_{p} \times L_{k}^{p}$ pre-coding and the $L_{k}^{p} \times L_{k}^{p}$ power allocation matrix of the PU transmitter respectively where $L_{k}^{p}$ is the number of the PU's parallel transmitted streams. The aforementioned abstracted formulation is done in the sense that the SU system receives a total signal without having any further information about the PU's transmission parameters. The indicator function $\mathbb{1}_{k}\{\cdot\}$ that appears in eq.(2) is defined as

$$
\mathbb{1}_{k}\left\{\mathbf{G}_{k} \mathbf{d}_{k n}\right\}=\left\{\begin{array}{ll}
\mathbf{G}_{l k} \mathbf{d}_{k n}, & \text { if PU is active } \\
\mathbf{0}_{R_{p}}, & \text { if the PU user is inactive }
\end{array},\right.
$$

Finally the received signal at an active PU in the $k-t h\left(K_{L} \leq\right.$ $\left.k \leq K_{L}+K_{U}\right)$ unlicensed band is given by

$$
\mathbf{r}_{k n}=\mathbf{G}_{k}^{\prime} \mathbf{d}_{k n}+\mathbf{F}_{k} \mathbf{S}_{l k} \sqrt{\mathbf{P}_{l k}} \mathbf{x}_{k n}+\mathbf{z}_{k n}^{\prime}
$$

where again for simplicity we have incorporated the channel, pre-coding and power allocation matrix of the PU transmission in matrix $\mathbf{G}^{\prime}{ }_{k}$. Matrix $\mathbf{F}_{k}$ is the $R_{p} \times T_{s}$ channel matrix from the LTE-A eNodeB to the receiver of the PU and $\mathbf{z}_{k n}^{\prime}$ is the corresponding noise vector at the PU receiver.

From the above description it is evident that the eNodeB has at its disposal a number of licensed and unlicensed bands in order to optimize the downlink communication to the available UEs in terms of the achievable overall sum rate. To that end the eNodeB should determine the optimal RB allocation among the UEs, the optimal pre- / post- coding matrices $\mathbf{T}_{l k}$ and $\mathbf{S}_{l k}$ and the optimal power allocation ones under the following constraints: 1) The eNodeB / UEs will generate/exhibit zero interference from / to the PU transmissions in the unlicensed bands, 2) Each UE can aggregate at most a number of $K_{\max }$ bands and 3) The transmissions are subject to a total transmission power constraint $P_{\max }$.

\section{Resource Allocation Problem}

Let us now proceed to the definition of the problem. The aim is to maximize the total sum rate of the downlink transmission subject to the enumerated constraints posed on the last paragraph of Sec. II. 
Thus, the following optimization problem should be solved

$$
\begin{aligned}
& \max _{\substack{\mathbf{T}_{l k}, \mathbf{S}_{l k}, \mathbf{P}_{l k}, \alpha_{l k} \\
\mathbf{p}_{l=1}}} \sum_{k=1}^{N_{U}} \sum_{k=1}^{K} \alpha_{l k} N_{c} \log _{2}\left(\left|\mathbf{I}_{R_{s}}+\frac{1}{\sigma_{z}^{2}} \mathbf{T}_{l k}^{H} \mathbf{H}_{l k} \mathbf{S}_{l k} \mathbf{P}_{l k} \mathbf{S}_{l k}^{H} \mathbf{H}_{l k}^{H} \mathbf{T}_{l k}\right|\right) \\
& \text { s.t. } \quad \mathbf{F}_{k} \mathbf{S}_{l k}=\mathbf{0} \quad \& \quad \mathbf{T}_{l k}^{H} \mathbf{G}_{l k}=\mathbf{0}, \quad \forall k \in \mathcal{K}_{\mathcal{A}} \& \forall 1 \leq l \leq N_{U} \\
& \sum_{l=1}^{N_{U}} \sum_{k=1}^{K} \alpha_{l k} \operatorname{tr}\left(\mathbf{P}_{l k}\right) \leq P_{\max } \quad \& \quad \mathbf{P}_{l k} \geq \mathbf{0} \\
& \sum_{k=1}^{K} \alpha_{l k} \leq 1 \quad \& \quad \sum_{l=1}^{N_{U}} \alpha_{l k} \leq K_{\max } \quad \& \quad \alpha_{l k} \in\{0,1\},
\end{aligned}
$$

where eq.(4) expresses the total sum rate of the downlink LTEA system, the binary variable $\alpha_{l k}$ is set to one when the $k-t h$ $\mathrm{RB}$ is allocated to the $l$-th user and zero if not, $\mathcal{K}_{\mathcal{A}} \subseteq\left[K_{L}+\right.$ $\left.1, K_{L}+K_{U}\right]$ is the set of the unlicensed bands with an active PU on them, $K=K_{L}+K_{U}$ and $\operatorname{tr}(\cdot)$ is the trace of a matrix. The constraints of eq.(5) are used to cancel the interference from/to the PUs by forcing the pre-coding matrix $\mathbf{S}_{l k}$ and the post-coding one to $\mathbf{T}_{l k}$ to be orthogonal to matrices $\mathbf{F}_{k}$ and $\mathbf{G}_{l k}$ respectively, according to eqs.(2)-(3).

Since the eNodeB handles exclusively the interference cancellation to the PU receivers by properly design of the its pre-coding matrix, the PU systems may continue to operate as if there is no interfering user in their band. Note also that the constraints of eq.(5) are active if at a PU user is active in the $\mathrm{RB}$ under consideration. The constraints of eq.(6) control the total transmission power and force the power allocation matrices to be positive respectively and the final ones of eqs.(7) are posed in order to control the maximum number of aggregated RBs per UE, the maximum number of allowed UEs per RB (1) and to force the specified support set on the parameters $\alpha_{l k}$ respectively.

\section{A. Problem Formulation}

Let us firstly consider the zero interference constraints in eq.(5). Let us assume that without loss of generality, matrices $\mathbf{S}_{l k}$ and $\mathbf{T}_{l k}$ can be decomposed as $\mathbf{S}_{l k}=\mathbf{Q}_{k} \mathbf{W}_{l k}$ and $\mathbf{T}_{l k}=$ $\mathbf{O}_{l k} \mathbf{B}_{l k}$ for the unlicensed RBs with an active PU on them. It is now straightforward to see that the zero interference constraints are equivalent to $\mathbf{F}_{k} \mathbf{Q}_{k}=\mathbf{0}$ and $\mathbf{O}_{l k}^{H} \mathbf{G}_{l k}=\mathbf{0}, \forall k \in \mathcal{K}_{\mathcal{A}}$, ones respectively. The previous constraints can be satisfied if matrices $\mathbf{G}_{l k}$ and $\mathbf{F}_{k}$ have a null-space which is equivalent to the case where the active PUs in the unlicensed band under consideration employ a number of spatial degrees of freedom $L_{k}^{p}$ that satisfies $L_{k}^{p}<\min \left\{R_{s}, T_{s}\right\}$. If the previous is not the case, this band cannot be employed by the UEs as the zero interference constraint cannot be satisfied and therefore we set $a_{l k}=0, \forall l \in\left[1, N_{U}\right]$ in the optimization problem of eqs.(4)-(7). On the other hand, if there is no active PU in the unlicensed band, there is also no need for interference cancellation and that unlicensed band is treated in a similar manner to the licensed ones. Therefore, if we define the set of the unlicensed bands with an active user of a non-zero rank null-space as $\mathcal{K}_{\mathcal{A}^{*}}$ we have that

$$
\mathbf{Q}_{k}=\mathcal{N}\left(\mathbf{F}_{k}\right) \quad \& \quad \mathbf{O}_{l k}=\mathcal{N}\left(\mathbf{G}_{l k}\right)
$$

and the optimization problem of eqs.(4)-(7) can be written in the following equivalent form

$$
\max _{\substack{\mathbf{T}_{l k}, \mathbf{S}_{l k}, \mathbf{P}_{l k}, \mathbf{S}_{l k}, \mathbf{B}_{l k}, \alpha_{l k} \\ \mathbf{W}_{l=1}}} \sum_{k \in \mathcal{K}_{\mathcal{F}}}^{N_{U}} \alpha_{l k} N_{c} \times
$$

$$
\begin{aligned}
& \log _{2}\left(\left|\mathbf{I}_{R_{x}}+\frac{1}{\sigma_{z}^{2}} \mathbf{T}_{l k}^{H} \mathbf{H}_{l k} \mathbf{S}_{l k} \mathbf{P}_{l k} \mathbf{S}_{l k}^{H} \mathbf{H}_{l k}^{H} \mathbf{T}_{l k}\right|\right)+ \\
& \sum_{l=1}^{N_{U}} \sum_{k \in \mathcal{K}_{\mathcal{A}^{*}}} \alpha_{l k} N_{c} \log _{2}\left(\left|\mathbf{I}_{R_{x}}+\frac{1}{\sigma_{z}^{2}} \mathbf{B}_{l k}^{H} \mathbf{J}_{l k} \mathbf{W}_{l k} \mathbf{P}_{l k} \mathbf{W}_{l k}^{H} \mathbf{J}_{l k}^{H} \mathbf{B}_{l k}\right|\right) \\
& \text { s.t. } \sum_{l=1}^{N_{U}} \sum_{k \in \mathcal{K}_{\mathcal{A}}^{*} \cup \mathcal{K}_{\mathcal{F}}} \alpha_{l k} \operatorname{tr}\left(\mathbf{P}_{l k}\right) \leq P_{\max } \quad \& \quad \mathbf{P}_{l k} \geq \mathbf{0} \text {, } \\
& \sum_{k \in \mathcal{K}_{\mathcal{A}^{*} \cup \mathcal{K}_{\mathcal{F}}}^{K}} \alpha_{l k} \leq 1 \quad \& \quad \sum_{l=1}^{N_{U}} \alpha_{l k} \leq K_{\max } \quad \& \quad \alpha_{l k} \in\{0,1\},
\end{aligned}
$$

where $\mathcal{K}_{\mathcal{F}}$ is the set that includes the licensed bands along with the free unlicensed RBs (the ones that are not occupied by an active PU) and matrix $\mathbf{J}_{l k}=\mathbf{O}_{l k}^{H} \mathbf{H}_{l k} \mathbf{Q}_{k}, \forall k \in \mathcal{K}_{\mathcal{A}^{*}}$ is the equivalent channel matrix in the unlicensed bands of that set.

In the optimization problem of eqs.(10)-(12) we have now two sets of sub-bands, set $\mathcal{K}_{\mathcal{F}}$ in which the UEs can employ all the available $\min \left\{R_{s}, T_{s}\right\}$ spatial degrees of freedom and the set $\mathcal{K}_{\mathcal{A}^{*}}$ where only the $\min \left\{R_{s}-L_{k}^{p}, T_{s}-L_{k}^{p}\right\}$ ones can be employed by considering the transmission through the equivalent MIMO system whose channel matrix is the $\mathbf{J}_{l k}$ one. With the latter transformation the LTE-A system satisfies both the zero interference constraints as it aligns its transmitted signals to orthogonal subspaces to the ones of the PUs at the unlicensed bands. Note that, given the orthonormal properties of matrices $\mathbf{O}_{l k}$ and $\mathbf{Q}_{l k}$ the statistics of the equivalent channel matrix $\mathbf{J}_{l k}$ is exactly the same with the ones of the original channel matrix $\mathbf{H}_{k n}$, and thus the only difference is on the dimensions of the equivalent MIMO system, since the latter are reduced by $L_{k}^{p}$ per dimension compared to the ones of the original system.

Now that the interference constraints are satisfied, we move forward in the derivation of the pre-/post coding matrices $\mathbf{S}_{l k}$, $\mathbf{W}_{l k}, \mathbf{T}_{l k}$ and $\mathbf{B}_{l k}$ of the equivalent optimization problem of eqs.(10)-(12). Let us recall that that in each one of the available RBs the LTE-A system can establish its communication link via a MIMO frequency flat fading channel whose channel matrix is either $\mathbf{H}_{l k}$ for licensed RBs and unlicensed RBs with no active PU or the equivalent one $\mathbf{J}_{l k}$ for an unlicensed $\mathrm{RB}$ with an active PU on it, as described in the previous paragraph. According to [19], a selection for pre-/post- coding matrices that maximizes the rate of such a system is based on the Singular Value Decomposition (SVD) of the corresponding channel matrix. That is,

$$
\begin{array}{rll}
\mathbf{S}_{l k}=\mathbf{V}_{l k} & \& & \mathbf{T}_{l k}=\mathbf{U}_{l k} \\
\mathbf{W}_{l k}=\tilde{\mathbf{V}}_{l k} & \& & \mathbf{B}_{l k}=\tilde{\mathbf{U}}_{l k},
\end{array}
$$

where $\mathbf{H}_{l k}=\mathbf{U}_{l k} \boldsymbol{\Sigma}_{l k} \mathbf{V}_{l k}^{H}, \forall k \in \mathcal{K}_{\mathcal{F}}$ and $\mathbf{J}_{l k}=\tilde{\mathbf{U}}_{l k} \tilde{\boldsymbol{\Sigma}}_{l k} \tilde{\mathbf{V}}_{l k}^{H}$, $\forall k \in \mathcal{K}_{\mathcal{A}^{*}}$.

Now by using the properties of the SVD and the ones of the determinant of a matrix on the cost function of the optimization problem of eqs.(10)-(12), we have

$$
\begin{aligned}
& \max _{\mathbf{P}_{l k}, \alpha_{l k}} \sum_{l=1}^{N_{U}} \sum_{k \in \mathcal{K}_{\mathcal{F}}} \alpha_{l k} \sum_{m=1}^{L^{s}} N_{c} \log _{2}\left(1+\frac{\mathbf{P}_{l k}(m, m) \boldsymbol{\Sigma}_{l k}^{2}(m, m)}{\sigma_{z}^{2}}\right)+ \\
& \sum_{l=1}^{N_{U}} \sum_{k \in \mathcal{K}_{\mathcal{A}^{*}}} \alpha_{l k} \sum_{m=1}^{L_{k}^{U}} N_{c} \log _{2}\left(1+\frac{\mathbf{P}_{l k}(m, m) \tilde{\boldsymbol{\Sigma}}_{l k}^{2}(m, m)}{\sigma_{z}^{2}}\right)
\end{aligned}
$$




$$
\begin{aligned}
& \text { s.t. } \quad \sum_{l=1}^{N_{U}} \sum_{k \in \mathcal{K}_{\mathcal{A}^{*} \cup \mathcal{K}_{\mathcal{F}}}} \alpha_{l k} t r\left(\mathbf{P}_{l k}\right) \leq P_{\max } \quad \& \quad \mathbf{P}_{l k} \geq \mathbf{0}
\end{aligned}
$$

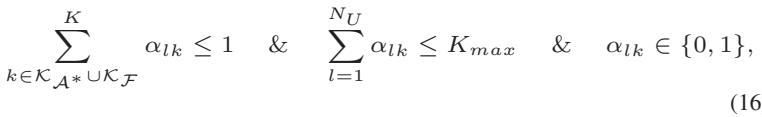

where $L^{s}=\min \left\{R_{s}, T_{s}\right\}$ and $L_{k}^{U}=\min \left\{R_{s}-L_{k}^{p}, T_{s}-L_{k}^{p}\right\}$. Thus, each RB is decomposed in a number of independent parallel channels. The optimization problem of eqs.(14)-(16) belongs to the class of Mixed Integer Non Linear Programming (MINLP) ones that are non-convex and NP hard to solve since its solution involves an exhaustive search on the $\alpha_{l k}$ values requiring $\mathcal{O}\left(K N_{U}^{K}\right)$ operations. To that end the optimal power allocation problem under consideration should be solved for any possible RB allocation in order to find the one that maximizes the overall system sum rate. In the related literature [20]-[21], several approaches are proposed for the solution of MINLP problems in the context of branch and bound algorithms or other known convex relaxation techniques. Several general purpose software solvers usually incorporate a method to solve MINLP problems, though the complexity still remains high. In the following subsection an alternative low complexity algorithm will be developed that achieves the optimal solution and thus it is possible to avoid the complexity of solving the MINLP problem.

\section{B. Proposed Solution}

In this subsection it is shown that the problem under consideration has an equivalent form that can be solved with less computational complexity than the MINLP one of eqs.(14)(16) by following a similar approach to the ones of [14]-[15]. Let us define the domain

$$
\begin{aligned}
& \mathcal{D}=\left\{\mathbf{P}_{l k} \geq 0 \mid \sum_{l=1}^{N_{U}} \mathbb{1}_{+}\left\{\mathbf{P}_{l k}\right\} \leq 1, \forall k \in[1, K] \&\right. \\
& \left.\sum_{k=1}^{N_{U}} \mathbb{1}_{+}\left\{\mathbf{P}_{l k}\right\} \leq K_{\text {max }}, \forall l \in\left[1, N_{U}\right]\right\},
\end{aligned}
$$

where the indicator functional $\mathbb{1}_{+}\{\cdot\}$ is one if at least one of the elements of matrix $\mathbf{P}_{l k}$ is non-zero and zero otherwise. For the set of power allocation matrices $\mathbf{P}_{l k} \in \mathcal{D}, 1 \leq l \leq$ $N_{U}, 1 \leq k \leq K$, denoted by $\left\{\mathbf{P}_{l k}\right\}$, the Lagrangian of the optimization problem of eqs.(14)-(16) can be written as

$$
\begin{aligned}
\mathcal{L}\left(\left\{\mathbf{P}_{l k}\right\}, \lambda\right) & =\sum_{l=1}^{N_{U}} \sum_{k \in \mathcal{K}_{\mathcal{F}}} \sum_{m=1}^{L^{s}} N_{c} \log _{2}\left(1+\frac{\mathbf{P}_{l k}(m, m) \boldsymbol{\Sigma}_{l k}^{2}(m, m)}{\sigma_{z}^{2}}\right)+ \\
& \sum_{l=1}^{N_{U}} \sum_{k \in \mathcal{K}_{\mathcal{A}^{*}}} \sum_{m=1}^{L_{k}^{U}} N_{c} \log _{2}\left(1+\frac{\mathbf{P}_{l k}(m, m) \tilde{\boldsymbol{\Sigma}}_{l k}^{2}(m, m)}{\sigma_{z}^{2}}\right)- \\
& \lambda\left(\sum_{l=1}^{N_{U}} \sum_{k \in \mathcal{K}_{\mathcal{A}^{*} \cup \mathcal{K}_{\mathcal{F}}}} \operatorname{tr}\left(\mathbf{P}_{l k}\right)-P_{\max }\right)
\end{aligned}
$$

where $\lambda \geq 0$ is the Lagrange multiplier. Then, the Lagrange dual function is given by

$$
g(\lambda)=\max _{\left\{\mathbf{P}_{l k}\right\} \in \mathcal{D}} \mathcal{L}\left(\left\{\mathbf{P}_{l k}\right\}, \lambda\right) .
$$

By inspection of eq.(19) one may see that the maximization of the Lagrangian function $\mathcal{L}\left(\left\{\mathbf{P}_{l k}\right\}\right)$ can be decomposed in to the following $K=\left|\mathcal{K}_{\mathcal{F}}\right|+\left|\mathcal{K}_{\mathcal{A}}^{*}\right|(|\cdot|$ is the cardinality of a set) simpler maximization problems

$$
\begin{aligned}
g_{k}^{A}(\lambda) & =\max _{\left\{\mathbf{P}_{l k}\right\} \in \mathcal{D}} \sum_{l=1}^{N_{U}} \sum_{m=1}^{L^{s}} N_{c} \log _{2}\left(1+\frac{\mathbf{P}_{l k}(m, m) \boldsymbol{\Sigma}_{l k}^{2}(m, m)}{\sigma_{z}^{2}}\right)- \\
& \lambda \sum_{l=1}^{N_{U}} \operatorname{tr}\left(\mathbf{P}_{l k}\right), \forall k \in \mathcal{K}_{\mathcal{F}} \\
g_{k}^{B}(\lambda) & =\max _{\left\{\mathbf{P}_{l k}\right\} \in \mathcal{D}} \sum_{l=1}^{N_{U}} \sum_{m=1}^{L_{k}^{U}} N_{c} \log _{2}\left(1+\frac{\mathbf{P}_{l k}(m, m) \tilde{\boldsymbol{\Sigma}}_{l k}^{2}(m, m)}{\sigma_{z}^{2}}\right)- \\
& \lambda \sum_{l=1}^{N_{U}} \operatorname{tr}\left(\mathbf{P}_{l k}\right), \forall k \in \mathcal{K}_{\mathcal{A}^{*}} .
\end{aligned}
$$

Then the Lagrange dual function can be written as

$$
g(\lambda)=\sum_{k \in \mathcal{K}_{\mathcal{F}}} g_{k}^{A}(\lambda)+\sum_{k \in \mathcal{K}_{\mathcal{A}^{*}}} g_{k}^{B}(\lambda)+\lambda P_{\text {max }} .
$$

Let us assume now that the $k$-th $\mathrm{RB}$ is allocated to the $l$-th user. For a fixed $\lambda$, the objective functions of eqs.(21)-(22) are concave ones with respect to $\mathbf{P}_{l k}$. For this case the optimal power allocation solution can be easily found to be

$$
\mathbf{P}_{l k}^{*}(m, m)=\left\{\begin{array}{l}
\left(\frac{N_{c}}{\ln (2) \lambda}-\frac{\sigma_{z}^{2}}{\Sigma_{l k}^{2}(m, m)}\right)^{+}, \forall \mathcal{K}_{\mathcal{F}} \\
\left(\frac{N_{c}}{\ln (2) \lambda}-\frac{\sigma_{z}^{2}}{\tilde{\boldsymbol{\Sigma}}_{l k}^{2}(m, m)}\right)^{+}, \forall \mathcal{K}_{\mathcal{A}^{*}}
\end{array}\right.
$$

where $(\cdot)^{+} \equiv \max \{\cdot, 0\}$. The solution to the optimization problem of eqs.(21)-(22) for the $k-t h \mathrm{RB}$ can be derived by evaluating the function $f_{l}^{A}\left(\lambda, \mathbf{P}_{l k}^{*}\right)$ if $k \in \mathcal{K}_{\mathcal{F}}$ or the function $f_{l}^{B}\left(\lambda, \mathbf{P}_{l k}^{*}\right)$ if $k \in \mathcal{K}_{\mathcal{A}^{*}}$,

$$
\begin{aligned}
f_{l}^{A}\left(\lambda, \mathbf{P}_{l k}^{*}\right)= & \sum_{m=1}^{L^{s}} N_{c} \log _{2}\left(1+\frac{\mathbf{P}_{l k}^{*}(m, m) \boldsymbol{\Sigma}_{l k}^{2}(m, m)}{\sigma_{z}^{2}}\right)- \\
\lambda & t r\left(\mathbf{P}_{l k}^{*}\right), \forall k \in \mathcal{K}_{\mathcal{F}} \\
f_{k}^{B}\left(\lambda, \mathbf{P}_{l k}^{*}\right)= & \sum_{m=1}^{L_{k}^{U}} N_{c} \log _{2}\left(1+\frac{\mathbf{P}_{l k}^{*}(m, m) \tilde{\boldsymbol{\Sigma}}_{l k}^{2}(m, m)}{\sigma_{z}^{2}}\right)- \\
\lambda \operatorname{tr}\left(\mathbf{P}_{l k}^{*}\right), \forall k \in \mathcal{K}_{\mathcal{A}^{*}} . &
\end{aligned}
$$

for each of one of the $N_{U}$ UEs and assigning the RB to UE that maximizes the corresponding function given that it has not yet reached the limit on the maximum number of allocated RBs $\left(K_{\max }\right)$. If this is the case, we need to consider the following cases.

1) If the UE achieves greater value for the eqs.(25)-(26) on the new RB than the minimum one achieved by the existing RBs then, the one that achieves the minimum value is replaced by the new RB. For the replaced RB we search in the set of the remaining UEs the one that maximizes eqs.(25)-(26) given that it has not also reached the limit on the maximum number of allocated RBs $\left(K_{\max }\right)$, repeating a similar case examination in an inductive way.

2) If the UE does not achieve greater value for the eqs.(25)(26) on the new RB than the minimum one achieved by the existing RBs then we move forward to the UE that achieves the second best value on the RB under consideration, and we examine the same cases again, in an inductive way.

Once eqs.(21)-(22) are solved for all $k$, the Lagrange dual 


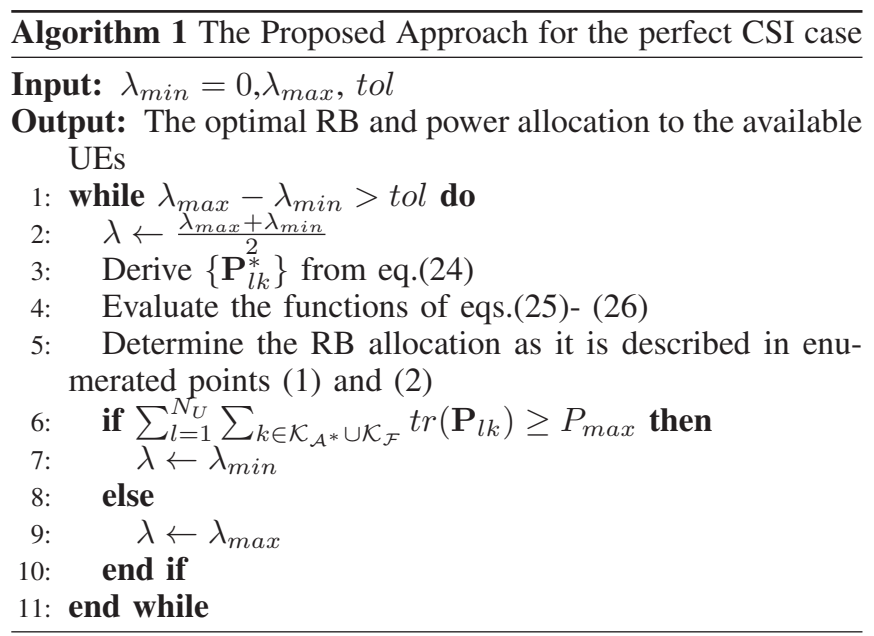

function can be derived from eq.(23) and the optimum value of the multiplier $\lambda^{*} \geq 0$ can be determined by applying a bisection method [22]. The complete procedure is summarized on Algorithm 1. By assuming that the values of the functions $f_{l}^{B}\left(\lambda, \mathbf{P}_{l k}^{*}\right)$ and $f_{l}^{B}\left(\lambda, \mathbf{P}_{l k}^{*}\right)$ of eqs.(25)-(26) are stored and sorted for each RB at each iteration of Algorithm 1, it is easy to see that the proposed approach requires $\mathcal{O}\left(K N_{U}\right)$ operations to find the optimal solution [15].

\section{Simulation Results}

In this section indicative simulations are provided in order to evaluate the performance of the proposed techniques. In Fig. 1 the performance in terms of the achievable data rate of the proposed approach is compared to the one of a LTE-A system that employs only a number $K_{L}$ fixed licensed bands for different SNR values. At first we examine the performance for $K_{L}=5$ licensed and $K_{U}=10$ unlicensed RBs. The PU and the LTE-A systems are assumed to be $2 \times 2$ and $4 \times 4$ MIMO ones respectively. The channel gains of all the involved channels are generated as circular symmetric i.i.d. Gaussian variables of zero mean and unit variance, i.e. $\mathcal{C N}(0,1)$. The performance is evaluated for two different PU activity scenarios $P_{k}=0.2$ and $P_{k}=0.8$. A two user system is assumed with the maximum permitted number of aggregated RBs $K_{\max }=5$. The solution to the licensed only case is given by solving the MINLP optimization problem of eqs.(4)(7) without the unlicensed bands constraint of eq.(5). The OPTI [23] optimization toolbox is used for the solution of all the MINLP problems considered here. The proposed approach ("MINLP $K_{L}=5, K_{U}=10, P_{k}=0.2$ " and "MINLP $K_{L}=5, K_{U}=10, P_{k}=0.8$ " curves) achieves significantly improved performance as compared to the licensed only case ("Licensed Only $N_{c}=0.5, N_{U}=10$ " curve). In the licensed only case two users share only $K_{L}=5 \mathrm{RBs}$ which is bellow their quota of $K_{\max }=5 \mathrm{RBs}$. Contrariwise in the unlicensed - licensed case there are $K_{L}+K_{U}=15$ available RBs so each one of the users can reach its quota by having also options for selecting the best possible combination of RBs that maximizes the sum rate of the system which explains the observed superiority in the performance. Note also that the proposed techniques achieve better performance for $P_{k}=0.2$ than for $P_{k}=0.8$. This can be explained by the fact that, for lower $P_{k}$ values there are unoccupied bands with higher

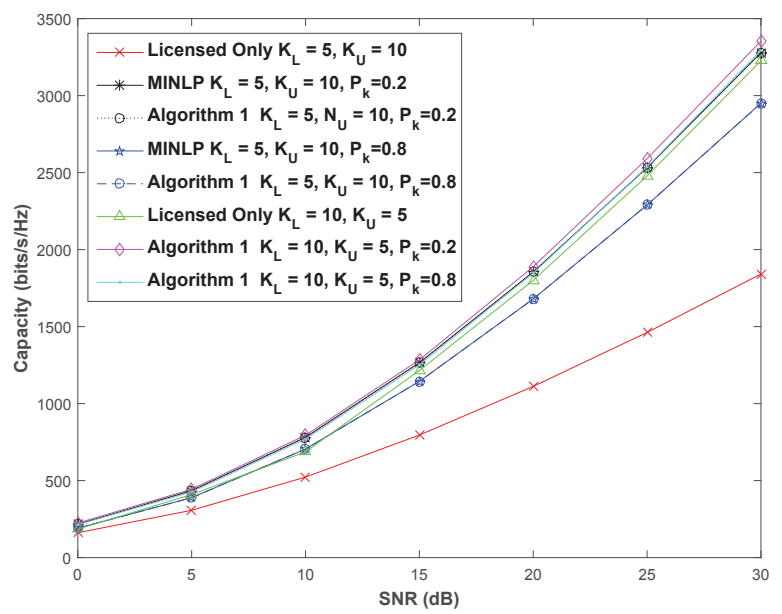

Fig. 1: Performance of the proposed approach under the perfect CSI case

probability. Thus, the LTE-A users have extra RBs without having to null out the interference from/to the PUs that leads to loss in spatial degrees of freedom as discussed in Sec.III. In the same figure the optimality of Algorithm 1 is verified as it achieves almost identical performance with the MINLP solutions ("Algorithm $1 K_{L}=5, K_{U}=10, P_{k}=0.2$ " and "Algorithm $1 K_{L}=5, K_{U}=10, P_{k}=0.8$ " curves).

We close the study by repeating the experiments of Algorithm 1 for the case of $K_{L}=10$ licensed and $K_{U}=5$ unlicensed RBs ("Algorithm $1 K_{L}=10, K_{U}=5, P_{k}=0.2$ " and "Algorithm $1 K_{L}=10, K_{U}=5, P_{k}=0.8$ " curves) and of the licensed only MINLP approach ("Licensed Only $N_{c}=0.5, N_{U}=10$ " curve). As we can see the performance gains here are smaller (but still existent) due to fact that the system has already $K_{L}=10$ available RBs which suffice for each user to reach its quota. Furthermore there are only 5 unlicensed RBs available, thus less available combinations to maximize the sum rate of the system. Of course with an increase in the number of available unlicensed RBs and/or LTE-A users, one will see a corresponding increase in the sum rate of the system.

\section{CONCLUSION}

In this paper, we considered the resource allocation problem for the licensed/unlicensed carrier aggregation MIMO scheme. We defined the problem at the downlink providing interference nulling, power allocation and RBs selection. Such a resource allocation problem is very critical in order to realize the licensed assisted access vision of 3GPP. The problem under consideration lies within the region of MINLP ones and therefore we were motivated to develop an optimal lowcomplexity solution based on the Lagrange dual decomposition approach. The performance of the proposed approach is verified by indicative simulations which highlight the gains of aggregating unlicensed bands and prove the optimality of the low-complexity proposed approach. Future works include the extension of the proposed approach in the uplink case and 
also in cases where alternative constraints (i.e. Qos) or cost functions (power minimization) are considered.

\section{ACKNOWLEDGMENT}

This work has been supported by the "Spectrum Overlay through Aggregation of Heterogeneous Dispersed Bands" project, ICT-SOLDER, www.ict-solder.eu, FP7 grant agree ment number 619687.

\section{REFERENCES}

[1] 3rd Generation Partnership Project (3GPP), "Evolved universal terrestrial radio access (e-utra) and evolved universal terrestrial radio access network (e-utran); overall description; stage 2," vol. TS 36.300, June 2012.

[2] — "TSG RAN; Study on Licensed-Assisted Access to Unlicensed Spectrum; rel.13; v13.0.0," vol. TR 36.889, June 2015.

[3] F. Liu, K. Zheng, W. Xiang, and H. Zhao, "Design and performance analysis of an energy-efficient uplink carrier aggregation scheme," IEEE Journal on Selected Areas in Communications, vol. 32, no. 2, pp. 197207, February 2014

[4] X. Lin, J. Andrews, and A. Ghosh, "Modeling, analysis and design for carrier aggregation in heterogeneous cellular networks," IEEE Trans. on Communications, vol. 61, no. 9, pp. 4002-4015, September 2013.

[5] R. Zhang, Z. Zheng, M. Wang, X. Shen, and L.-L. Xie, "Equivalent capacity in carrier aggregation-based lte-a systems: A probabilistic analysis," IEEE Trans. on Wireless Communications, vol. 13, no. 11, pp. 6444-6460, Nov 2014

[6] S. Lim, G. Noh, H. Kim, and D. Hong, "Optimal tone space selection scheme for ofdma-vts in carrier aggregation," IEEE Trans. on Wireless Communications, vol. 12, no. 11, pp. 5679-5691, November 2013.

[7] R. Zhang, M. Wang, Z. Zheng, X. Shen, and L.-L. Xie, "Crosslayer carrier selection and power control for lte-a uplink with carrier aggregation," in Global Communications Conference (GLOBECOM), 2013 IEEE, Dec 2013, pp. 4668-4673.

[8] H.-S. Liao, P.-Y. Chen, and W.-T. Chen, "An efficient downlink radio resource allocation with carrier aggregation in lte-advanced networks," Mobile Computing, IEEE Transactions on, vol. 13, no. 10, pp. 22292239, Oct 2014

[9] K. Werner, H. Asplund, B. Halvarsson, A. Kathrein, N. Jalden, and D. Figueiredo, "Lte-a field measurements: $8 \times 8$ mimo and carrier aggregation," in Vehicular Technology Conference (VTC Spring), 2013 IEEE 77th, June 2013, pp. 1-5.

[10] Y. Kakishima, K. Takeda, T. Kawamura, Y. Kishiyama, H. Taoka, and T. Nakamura, "Experimental evaluations on carrier aggregation and multi-user mimo associated with evd-based csi feedback for lteadvanced downlink," in Wireless Communication Systems (ISWCS), 2011 8th International Symposium on, Nov 2011, pp. 884-888.

[11] A. Cattoni, H. Nguyen, J. Duplicy, D. Tandur, B. Badic, R. Balraj, F. Kaltenberger, I. Latif, A. Bhamri, G. Vivier, I. Kovacs, and P. Horvath, "Multi-user mimo and carrier aggregation in 4g systems: The samurai approach," in IEEE Wireless Communications and Networking Conference Workshops (WCNCW), April 2012, pp. 288-293.

[12] A. Bhamri, F. Kaltenberger, R. Knopp, and J. Hamalainen, "Improving mu-mimo performance in lte-(advanced) by efficiently exploiting feedback resources and through dynamic scheduling," in IEEE Wireless Communications and Networking Conference (WCNC), April 2013, pp. 563-567.

[13] F. Kaltenberger and S. Wagner, "Experimental analysis of networkaided interference-aware receiver for lte mu-mimo," in 8th IEEE Sensor Array and Multichannel Signal Processing Workshop (SAM), 2014, June 2014, pp. 325-328.

[14] W. Yu and R. Lui, "Dual methods for nonconvex spectrum optimization of multicarrier systems," IEEE Trans. on Communications, vol. 54, no. 7, pp. 1310-1322, July 2006.

[15] K. Seong, M. Mohseni, and J. Cioffi, "Optimal resource allocation for ofdma downlink systems," in IEEE International Symposium on Information Theory, July 2006, pp. 1394-1398.
[16] Y. Zhang and C. Leung, "Resource allocation in an ofdm-based cognitive radio system," Communications, IEEE Transactions on, vol. 57, no. 7, pp. 1928-1931, July 2009.

[17] S. Almalfouh and G. Stuber, "Interference-aware radio resource allocation in ofdma-based cognitive radio networks," IEEE Trans. on Vehicular Technology, vol. 60, no. 4, pp. 1699-1713, May 2011.

[18] R. Zhang, M. Wang, L. Cai, Z. Zheng, and X. Shen, "Lte-unlicensed: the future of spectrum aggregation for cellular networks," IEEE Wireless Communications, vol. 22, no. 3, pp. 150-159, June 2015

[19] D. Tse and P.Viswanath, Fundamentals of Wireless Communications. Cambridge University Press, 2004.

[20] B. Borchers and J. E. Mitchell, "An improved branch and bound algorithm for mixed integer nonlinear programs," Computers \& Operations Research, vol. 21, no. 4, pp. 359-367, 1994.

[21] _ - "An improved branch and bound algorithm for mixed integer nonlinear programs," Computers \& Operations Research, vol. 21, no. 4 pp. 359-367, 1994.

[22] S. Boyd and L. Vandenberghe, Convex Optimization. New York, NY, USA: Cambridge University Press, 2004.

[23] “Opti toolbox," lhttp://www.i2c2.aut.ac.nz/Wiki/OPTI/index.php 